\title{
The LBTI Fizeau imager - I. Fundamental gain in high-contrast imaging
}

\author{
F. Patru, ${ }^{1,2 \star}$ S. Esposito, ${ }^{2}$ A. Puglisi, ${ }^{2}$ A. Riccardi, ${ }^{2}$ E. Pinna, ${ }^{2}$ C. Arcidiacono, ${ }^{3}$ \\ J. Antichi ${ }^{2,4}$ B. Mennesson, ${ }^{5}$ D. Defrère, ${ }^{6,7}$ P. M. Hinz ${ }^{7}$ and J. M. Hill ${ }^{7}$ \\ ${ }^{1}$ PSL Research University, CNRS, Sorbonne Universités, UPMC Univ. Paris 06, Univ. Paris Diderot, Sorbonne Paris Cité, Observatoire de Paris, LESIA, 5 \\ place Jules Janssen, F-92195 Meudon cedex, France \\ ${ }^{2}$ INAF Osservatorio Astrofisico di Arcetri, 5 Largo Enrico Fermi, I-50125 Firenze, Italy \\ ${ }^{3}$ INAF Osservatorio Astronomico di Bologna, 1 Via Ranzani, I-40127 Bologna, Italy \\ ${ }^{4}$ Airbus Defense and Space GmbH, Space Systems, Robert Koch Str. 1 - D-82024 Taufkirchen, Munich, Germany \\ ${ }^{5}$ Jet Propulsion Laboratory, 4800 Oak Grove Drive, Pasadena, CA 91109, USA \\ ${ }^{6}$ Space Sciences, Technologies and Astrophysics Research Institute, University of Liége, 7 place du 20-Aožt, B-4000 Liége, Belgium \\ ${ }^{7}$ Steward Observatory, University of Arizona, 933 N. Cherry Avenue, Tucson, AZ 85721, USA
}

Accepted 2017 July 28. Received 2017 July 26; in original form 2017 April 5

\begin{abstract}
We show by numerical simulations a fundamental gain in contrast when combining coherently monochromatic light from two adaptive optics (AO) telescopes instead of using a single stand-alone AO telescope, assuming efficient control and acquisition systems at high speed. A contrast gain map is defined as the normalized point spread functions (PSFs) ratio of a single Large Binocular Telescope (LBT) aperture over the dual Large Binocular Telescope Interferometer (LBTI) aperture in Fizeau mode. The global gain averaged across the AO-corrected field of view is improved by a factor of 2 in contrast in long exposures and by a factor of 10 in contrast in short exposures (i.e. in exposures, respectively, longer or shorter than the coherence time). The fringed speckle halo in short exposures contains not only highangular resolution information, as stated by speckle imaging and speckle interferometry, but also high-contrast imaging information. A high-gain zone is further produced in the valleys of the PSF formed by the dark Airy rings and/or the dark fringes. Earth rotation allows us to exploit various areas in the contrast gain map. A huge-contrast gain in narrow zones can be achieved when both a dark fringe and a dark ring overlap on to an exoplanet. Compared to a single 8-m LBT aperture, the 23-m LBTI Fizeau imager can provide a gain in sensitivity (by a factor of 4), a gain in angular resolution (by a factor of 3 ) and, as well, a gain in raw contrast (by a factor of 2-1000 varying over the AO-corrected field of view).
\end{abstract}

Key words: instrumentation: adaptive optics - instrumentation: interferometers-methods: numerical-techniques: high angular resolution-techniques: interferometric.

\section{INTRODUCTION}

The LBTI (Large Binocular Telescope Interferometer; Angel \& Woolf 1997; Angel et al. 1998; Herbst 2003; Hill et al. 2014; Hinz et al. 2014, 2016) used in Fizeau mode can produce direct images by combining coherently the beams separated by a 14.4-m baseline from the two 8.4-m primary mirrors of the LBT (Large Binocular Telescope). Fizeau imaging can deliver the sensitivity of a $12-\mathrm{m}$ telescope and the angular resolution of a 23-m telescope across the field of view provided by two adaptive optics (AO) systems. We show in this paper that, compared to a single stand-alone LBT aperture, the LBTI Fizeau imager can also significantly improve the raw contrast by means of two first light adaptive optics (FLAO)

^E-mail: fabienpatru@gmail.com systems (Esposito et al. 2010). The LBTI is able to provide fringes since 2010, complemented with AO-correction since 2012 (Hinz et al. 2012; Leisenring et al. 2012) and with piston compensation in closed loop using group delay tracking since 2013 (Defrère et al. 2014).

We have performed here numerical simulations for the LBTI Fizeau imager including opto-dynamical aberrations, due here to AO errors, while other errors have been neglected. For each frame, two independent FLAO phase screens - generated by PASSATA (Agapito, Puglisi \& Esposito 2016) - are introduced in the complex pupil function reproducing realistic injection of the two separated beams into the beam combiner. Given an incoming wavefront with aberrations, we deduce the imaging properties by computing the point spread function (PSF) for short or long exposures. Those simulations have been described more in details in previous papers (Patru et al. 2016a,b). 

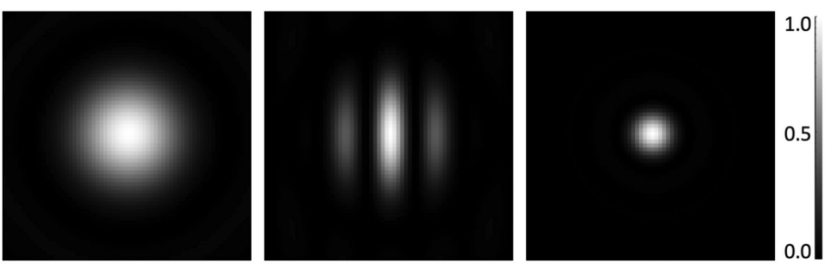

Figure 1. Theoretical PSF distributions of the 8-m LBT telescope (left), the 23-m LBTI interferometer (middle) and a 23-m telescope (right). Linear scale.

In this study, we consider no additional errors between the two LBT subapertures (e.g. residual piston, tip-tilt). We assume favourable conditions of observations with a good seeing and negligible atmospheric effects between the two telescopes. Actually, the atmospheric conditions across a large turbulent cell of $23 \mathrm{~m}$ of width feeding the two 8-m telescopes will produce differential piston and tip-tilt effects (Patru et al. 2017). We consider that the LBTI facility is equipped with efficient FLAO systems at high Strehl and a fast piston-tip-tilt mirror. We assume that the differential piston and tip-tilt induced by the atmosphere is well corrected by the fast mirror. Knowing that the main error of an AO system is, by far, the residual tip-tilt (Noll 1976), we consider here only the low differential tip-tilt due to the residual AO errors at high Strehl. It mainly affects the outer part of the field of view but not at the centre. We neglect any other differential wavefront errors that may occur at the LBTI facility (e.g. pointing errors, vibrations, misalignments).

We have performed monochromatic calculations at a single visible wavelength $(\lambda=750 \mathrm{~nm})$, but it can be applied to any observing wavelength. The effects of the spectral bandwidth and other instrumental aberrations are beyond the scope of this study. Despite all those assumptions, the results presented here are relevant for a high-Strehl AO regime in good atmospheric conditions providing narrow-band filters. In a future paper, we will develop an end-to-end simulation tool by modelling science cases and realistic aberrations and by using post-processing techniques.

In this paper, we compare the PSFs provided by a single LBT telescope and by the LBTI Fizeau interferometer (Section 2) showing a gain in contrast when combining coherently the two subapertures instead of using them individually (Section 3). We define a contrast gain map as the ratio of the PSFs provided by the LBTI versus the LBT (Section 3.1). We estimate the detectability of an exoplanet in the PSF by means of Earth rotation by taking advantage of the numerous high-contrast gain zones in the AO-corrected field of view (Section 3.2). We finally discuss how the LBTI Fizeau imager can improve the contrast in comparison with homologous telescopes made of a single aperture (Section 4).

\section{IMAGING PROPERTIES OF THE LBTI VERSUS THE LBT}

\subsection{Theoretical PSF}

While the PSF of a single LBT aperture is close to an Airy pattern, the PSF of the LBTI is made of rings (diffraction Airy pattern provided by each subaperture) and fringes (interference cosine pattern provided by both subapertures) that are alternatively bright or dark (Patru et al. 2017, Figs 1 and 2). The bright rings and fringes are features located on to a side-lobe in the PSF profile, where the high irradiance mitigates the achievable contrast. The dark rings and fringes are located into a valley (between two side-lobes), where the low irradiance enables locally a high contrast. A huge contrast in narrow zones can be achieved when both a dark fringe and a dark ring overlap.

The theoretical PSF contains fringes in both the Airy disc and Airy rings everywhere in the AO-corrected field of view (Figs 2 and 4). The LBTI provides a theoretical contrast exceeding the one of a single 8-m LBT and reaching the one of a 23-m telescope in some areas of the PSF. The angular resolution attains $\lambda / B_{\max }=6.8$ mas at $\lambda=750 \mathrm{~nm}$ in the longest $23-\mathrm{m}$ baseline direction. In the perpendicular direction crossing an LBT aperture of diameter $D=8.4$, the degenerated PSF profile yields to a resolution of $\lambda / D=18.4$ mas reduced by a factor of $B_{\max } / D \approx 3$ (Figs 1 and 2 ).

The PSF irradiance reaches its theoretical maximum as long as the interference pattern and the two diffraction patterns are perfectly centred on to the same pointing direction (McCarthy et al. 2000). The maximum irradiance is increased by a factor of 4 when combining coherently the two subapertures instead of using them individually. First, the entrance aperture collects two times more flux producing two superimposed Airy patterns in the PSF. Secondly, the interferometry states that the energy transferred from the dark fringes into the bright fringes intensifies the maximum of the fringes by a factor of 2 .

\subsection{Aberrated PSF}

We compare the PSFs of the LBT and the LBTI (Figs 4 and 5) in the presence of residual aberrations in the complex amplitude (Fig. 3). A temporal sequence of wavefronts is computed with phase rms errors of $\approx 100 \mathrm{~nm}$ (i.e. AO Strehl of 85 per cent in $H$ band and 50 per cent in $I^{\prime}$ band). In the presence of residual AO aberrations, the diffraction-limited PSF, made of a fringed Airy pattern, reduces to an AO-corrected PSF, made of a central core surrounded by a speckle halo (Fig. 4). The achievable Strehl by the LBTI using two AO is close to the Strehl provided by a single stand-alone AO system, as long as other differential wavefront errors can be mitigated. In practice, the LBTI Fizeau imager is primarily limited by the AO performance and, as well, by the differential piston/tiptilt errors between the two LBT apertures (Patru et al. 2017).

The LBTI Fizeau imager can be used for high-contrast imaging, providing a high-Strehl regime (by both AO systems), a cophasing mode (by a fringe tracker) and a burst mode (by a fast camera) to record speckles in short exposures (Patru et al. 2017). A raw gain in contrast is achieved when combining coherently the two subapertures instead of using them individually (Fig. 5).

\section{CONTRAST GAIN OF THE LBTI VERSUS THE LB T}

\subsection{Contrast gain map definition}

We define next a contrast gain map as the normalized PSFs ratio of a single stand-alone LBT aperture over the dual LBTI aperture in Fizeau mode. The raw contrast gain $G(x, y)$ performed by the dual versus single aperture is computed for each local position $(x$, y) across the AO-corrected field of view,

$G(x, y)=\frac{\operatorname{PSF}_{\mathrm{LBT}} / \operatorname{PSF}_{\mathrm{LBT}}^{\mathrm{Th}}(0,0)}{\mathrm{PSF}_{\mathrm{LBTI}} / \operatorname{PSF}_{\mathrm{LBTI}}^{\mathrm{Th}}(0,0)}=4 \cdot \frac{\mathrm{PSF}_{\mathrm{LBT}}}{\mathrm{PSF}_{\mathrm{LBTI}}}$

This map is normalized so that the contrast gain and the PSFs maxima equal to 1 for the diffraction-limited case. The factor 4 

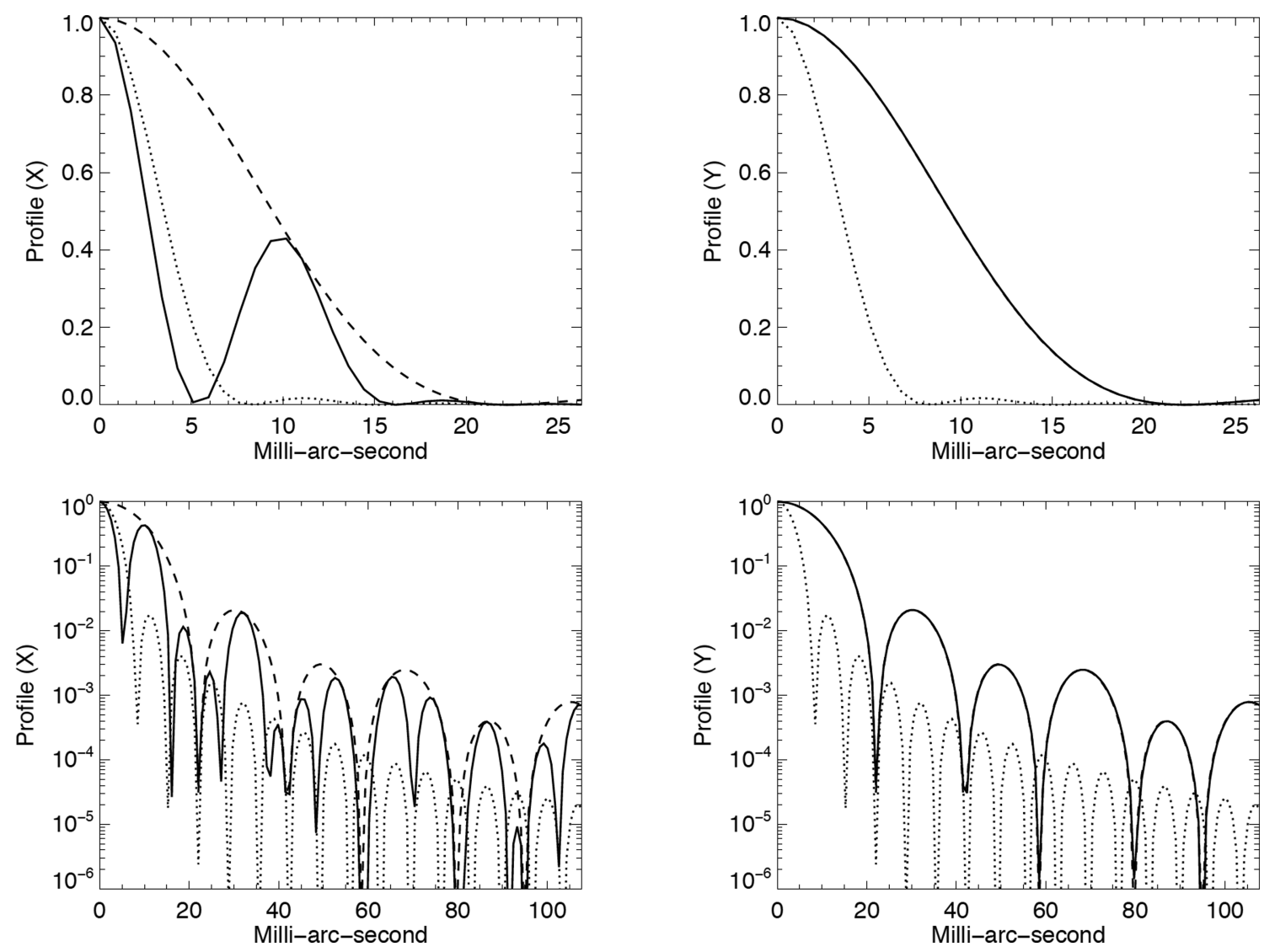

Figure 2. Theoretical PSF profiles of the 8-m LBT telescope (dashed line), the 23-m LBTI interferometer (solid line) and a 23-m telescope (dotted line), in the $X$ and $Y$ directions, respectively, parallel (left) and perpendicular (right) to the baseline of the LBTI. Linear (top) and logarithmic (bottom) scale.
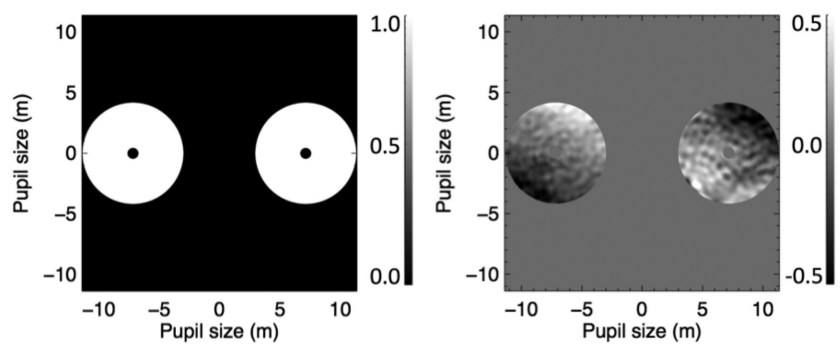

Figure 3. Complex amplitude of the LBTI with a uniform illumination in amplitude (left) and phase rms errors of about $\lambda / 8 \approx 100 \mathrm{~nm}$ (right), assuming no differential errors (e.g. piston, tip-tilt) between the two LBT apertures. Aberrations in $\mu \mathrm{m}$.

accounts for the double collected flux and the fringe intensification (Section 2.1).

We show contrast gain maps (Fig. 6) and their profiles (Fig. 7). We distinguish the ratio of diffraction-limited exposures, the ratio of short exposures, the ratio of long exposures $\left[G_{\mathrm{LE}}(x, y)\right]$ and the average of the ratios of short exposures $\left[G_{\mathrm{SE}}(x, y)\right]$. Black and white in the maps mean, respectively, no gain and a gain higher than 100 . A gain of about 10 is produced in the dark Airy rings, yielding to grey circles in the map. A gain above 100 can even be reached in the dark fringes, yielding to vertical white lines in the map. Continuous white lines appear in the diffraction-limited case corresponding to the well-contrasted fringes. The contrast gain occurs in the valleys of the LBTI PSF formed by the dark rings and fringes. The contrast is more favourable if the science object is located in a dark fringe
$(G \approx 10-100)$ or in a dark $\operatorname{ring}(G \approx 4-20)$, while it is not improved in the bright rings and fringes $(G \approx 1)$.

The gain averaged across the $\mathrm{AO}$-corrected field of view equals to $G_{\mathrm{LE}} \approx 2$ and $G_{\mathrm{SE}} \approx 10$ (Fig. 7). The global contrast is thus increased by a factor of 2 in long exposures and by a factor of 10 in short exposures (i.e. in exposures, respectively, longer or shorter than the coherence time). The contrast gain remains significant across the AO-corrected field of view for the average of the ratios of short exposures. Indeed, both fringes and speckles are frozen and remain contrasted during snapshots, contrary to long exposures where the fringes are blurred (Fig. 4). The fringed speckles in short exposures still contain high-angular resolution and high-dynamic range, enabling to resolve and highlight small and contrasted features of the science object.

\subsection{Contrast gain versus parallactic angle and radial distance}

We transpose this map as a function of the parallactic angle and of the radial distance from the axis to evaluate the exoplanet detection as a science case. Indeed, Earth rotation enables to probe many areas in the contrast gain map for searching extrasolar planets. Therefore, the LBTI Fizeau imager suits well to the technique of Angular Differential Imaging (ADI; Marois et al. 2006), taking advantage of the numerous high-contrast gain zones where an exoplanet can emerge from the fringed speckle halo. The aim of the following simulation is simply to estimate the detectability of an exoplanet in the PSF by means of Earth rotation.

Due to the alt-azimuth mount of the LBTI, the two primary mirrors remain horizontal while the fringes remain perpendicular to 

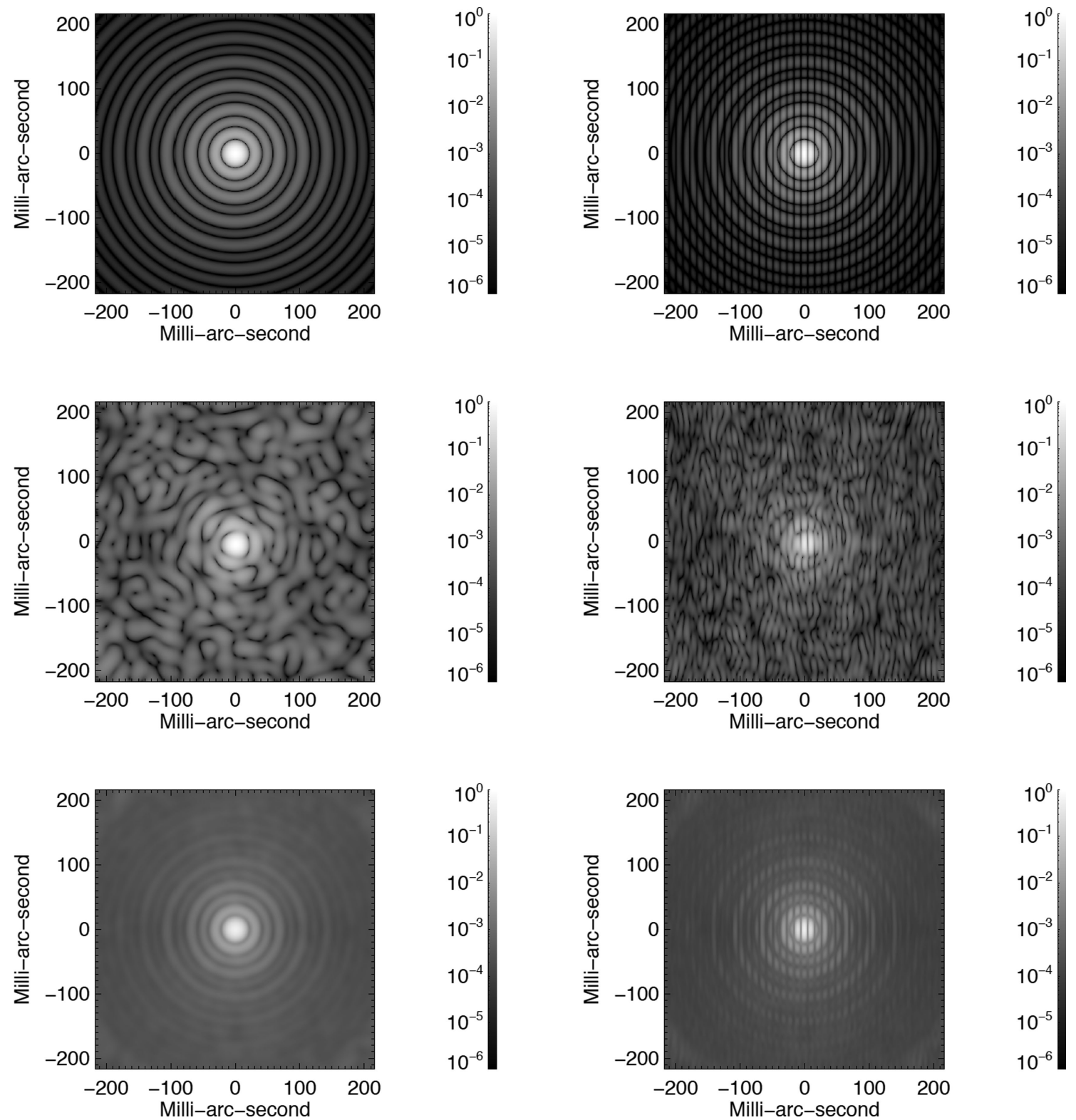

Figure 4. PSF distributions of a single LBT aperture (left) and the dual LBTI aperture (right). Diffraction-limited exposure (top), AO-corrected short exposure (middle) and long exposure (bottom). Monochromatic light at $\lambda=750 \mathrm{~nm}$.
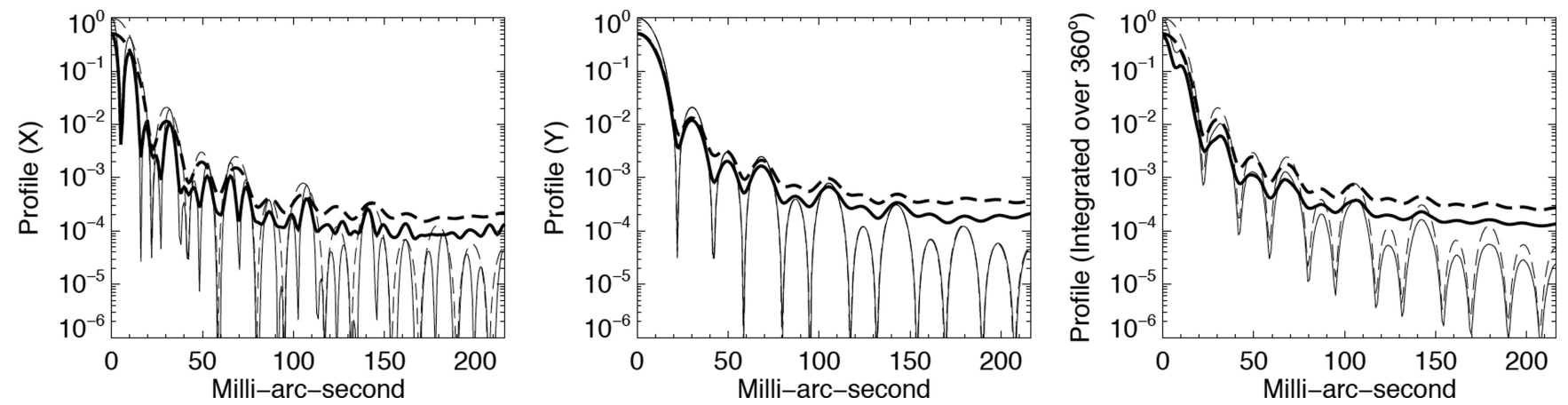

Figure 5. PSF profiles of a single LBT aperture (dashed line) and the dual LBTI aperture (solid line). Diffraction-limited exposure (thin line) and AO-corrected long exposure (bold line). Profile in the $X$ and $Y$ directions, respectively, parallel (left) and perpendicular (middle) to the baseline of the LBTI and profile integrated over $360^{\circ}$ (right). 

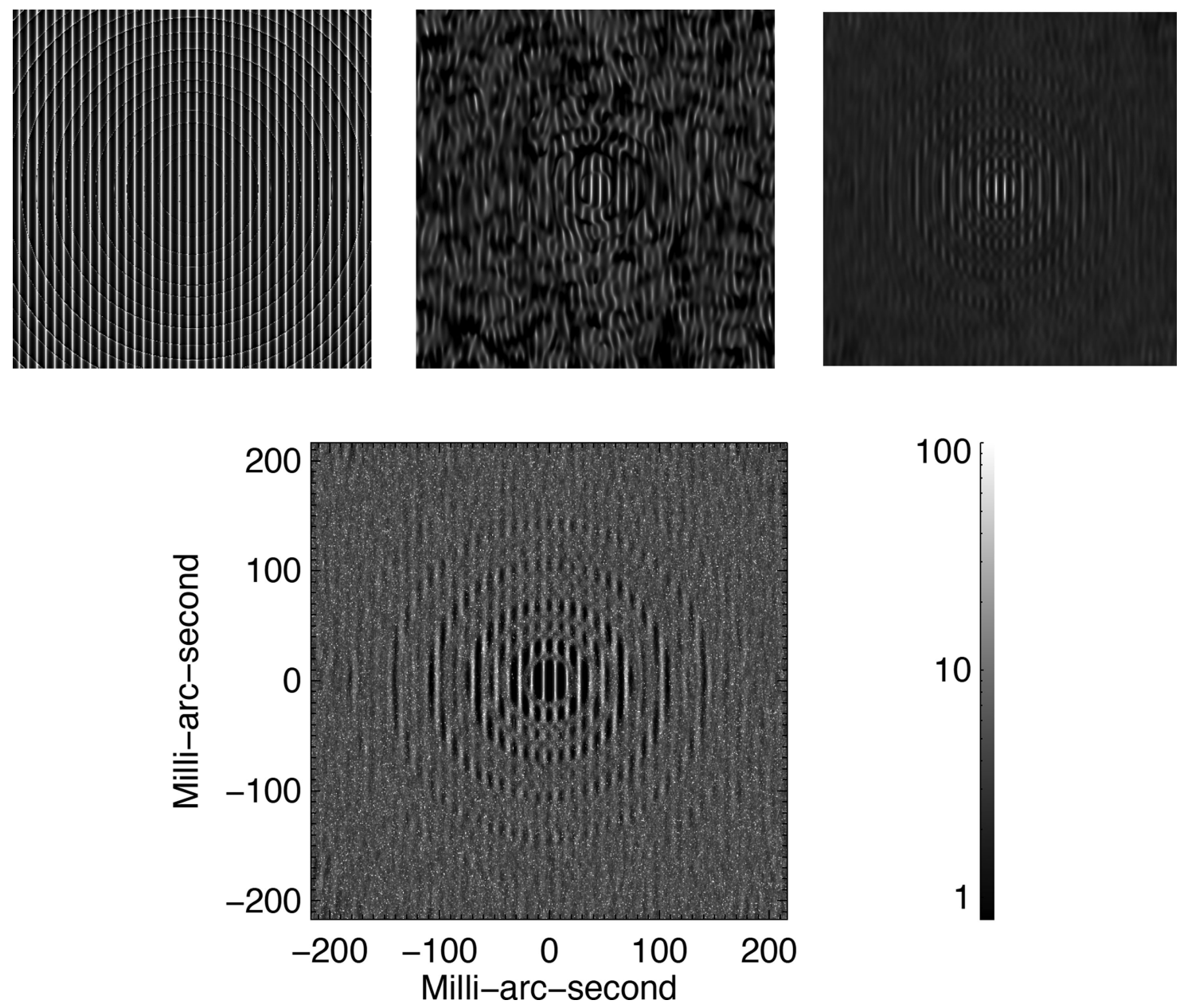

Figure 6. Contrast gain maps of a single LBT aperture over the dual LBTI aperture. Diffraction-limited exposures ratio (top left), short exposures ratio (top middle), long exposures ratio $G_{\mathrm{LE}}$ (top right) and averaged short exposures ratio $G_{\mathrm{SE}}$ (bottom). Monochromatic light at $\lambda=750 \mathrm{~nm}$.
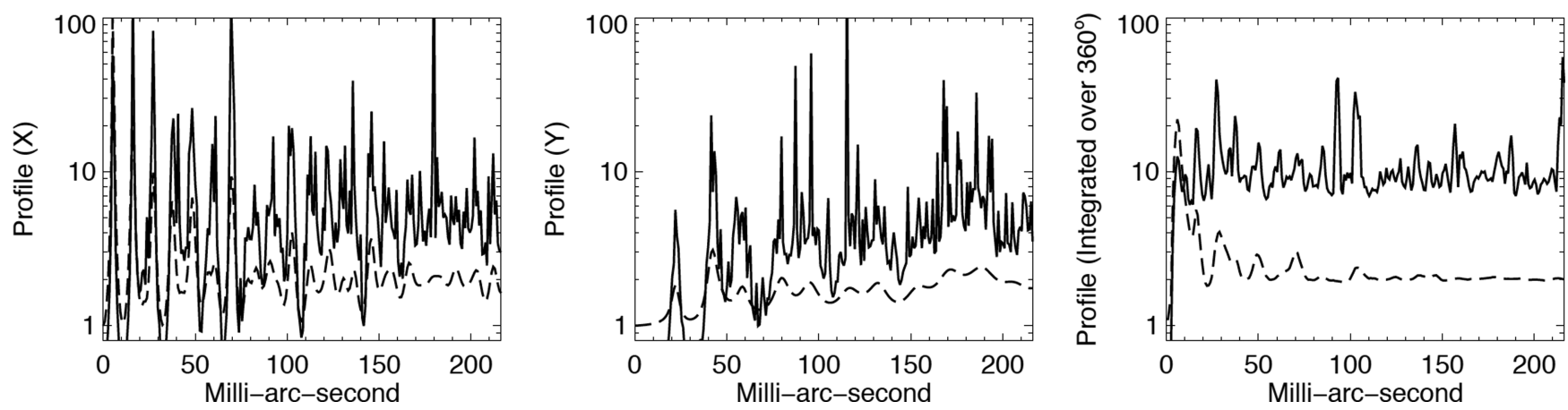

Figure 7. Contrast gain profiles of a single LBT aperture over the dual LBTI aperture. Long exposures ratio $G_{\mathrm{LE}}$ (dashed line) and averaged short exposures ratio $G_{\mathrm{SE}}$ (solid line). Profile in the $X$ and $Y$ directions, respectively, parallel (left) and perpendicular (middle) to the baseline of the LBTI and profile integrated over $360^{\circ}$ (right).

the horizon and fixed on the scientific detector. Since the sky rotates with respect to the fixed entrance pupil, an exoplanet at a given separation describes a circular curve around the PSF centre as a function of its parallactic angle. The field rotation moves an exoplanet through several high-gain zones. The field rotation performed by the LBTI is recommended for calibrating purpose with high-contrast imaging techniques (e.g. ADI).

In the next simulations, considering an instrument free of static aberration (without non-common path aberrations and quasi-static speckles), we assume the equivalence between pupil stabilized mode and field stabilized mode. Moving the star along a circle in a fixed PSF or a fixed map is here equivalent to maintaining the star at a fixed location while rotating the PSF or the map around their centre.

A contrast gain map (Fig. 6) has been circularly integrated by rotating it around the central axis (Fig. 8) and by assuming a uniform rotation rate. While fringes do not rotate in the case of the LBTI, Fig. 8 shows the sky coverage where a high contrast is achieved 

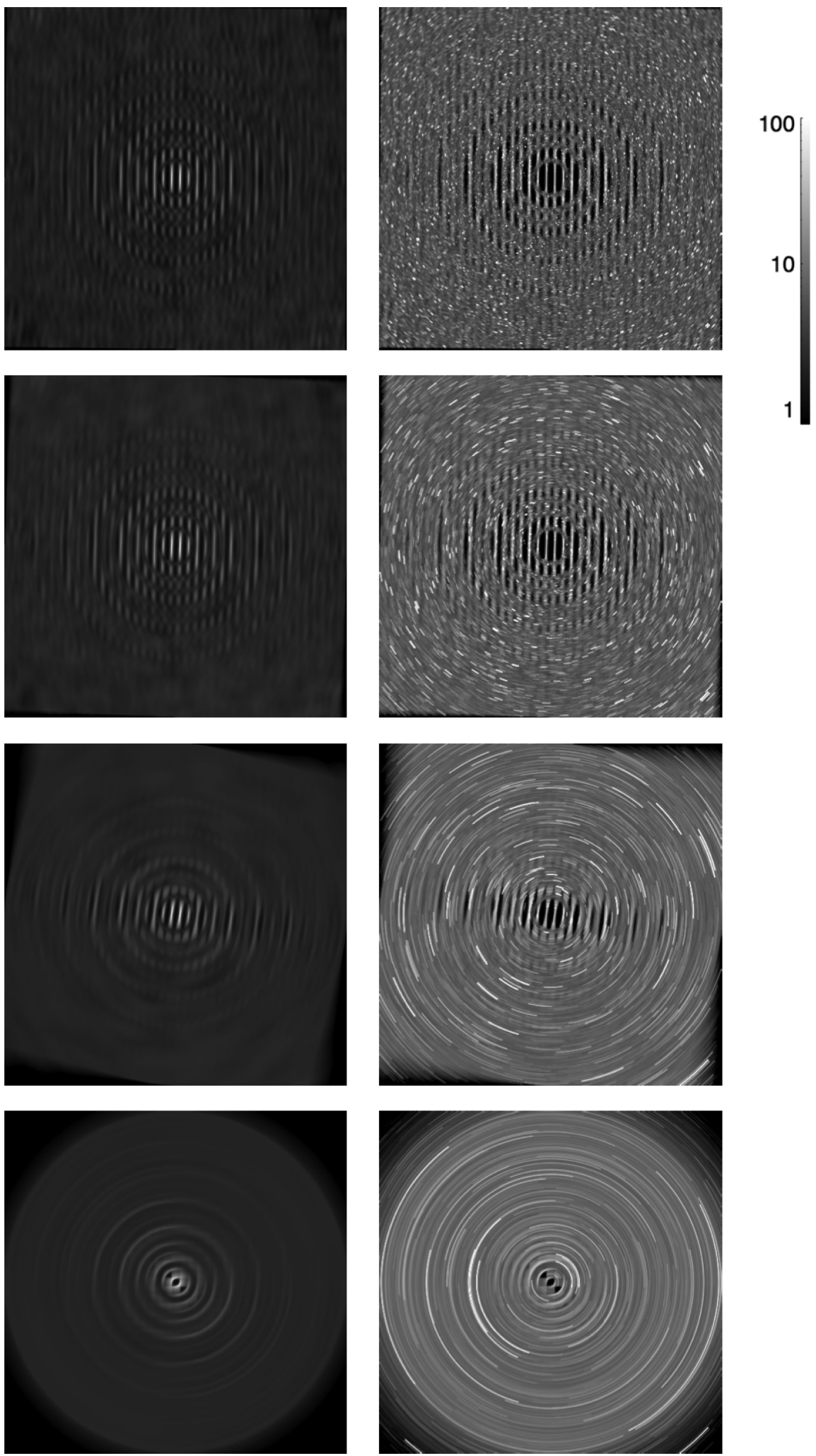

Figure 8. Circularly integrated contrast gain maps of the dual versus single aperture uniformly rotated over $1^{\circ}, 2^{\circ}, 15^{\circ}$ and $90^{\circ}$ (top to bottom). Long exposures ratio $G_{\mathrm{LE}}$ (left) and averaged short exposures ratio $G_{\mathrm{SE}}$ (right). Same scale as in Fig. 6 .

during an observation as a function of the rotation angle. After $15^{\circ}$ of rotation, one or few high-gain zones are crossed by the science object, whatever its position is in the field of view. Such constraint is mitigated by observing stars further away, whereas a higher parallactic rotation rate is required for science objects at low separation.

We show next a contrast gain map as a function of the radial distance and the parallactic angle (Fig. 9). To compute such map, 

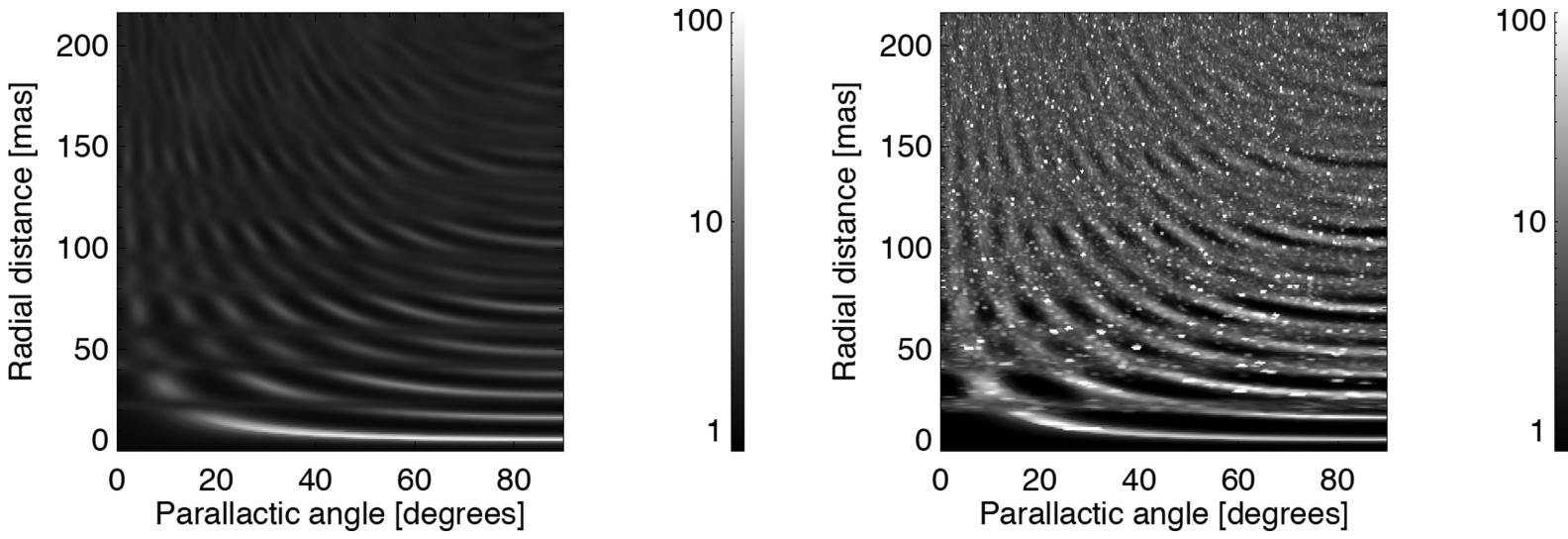

Figure 9. Contrast gain maps of the dual versus single aperture depending on the radial distance and the parallactic angle over $90^{\circ}$. Long exposures ratio $G_{\mathrm{LE}}$ (left) and averaged short exposures ratio $G_{\mathrm{SE}}$ (right).

each one of the circularly integrated images (Fig. 8) has been stacked into a data cube. Next, a layer in the data cube perpendicular to the $(x, y)$ plan and passing through its centre along the parallactic angle axis has been rotated and added for both short or long exposures. Finally, we obtain the profiles of the contrast gain depending on the parallactic angle at several radial distances (Fig. 10) by crossing the map in Fig. 9 along horizontal lines.

During the observation, the contrast gain evolves when the exoplanet moves circularly around the PSF centre at a given separation (Fig. 10). Let us consider short exposures that provide a higher contrast gain than long exposures, not only in average but also in some locations where dark fringes and rings intensify significantly the gain. An exoplanet is either located in a bright ring $(G \approx 1)$ or in a dark ring $(G \approx 4-20)$, depending on its radial distance. An exoplanet will be alternatively located in a bright fringe $(G \approx$ $1)$ or in a dark fringe $(G \approx 10-100)$ depending on the parallactic angle (Figs 9 and 10$)$. A huge-contrast gain $(G \approx 100-1000)$ in narrow zones (few milliarcseconds) can be achieved when both a dark fringe and a dark ring overlap on to an exoplanet.

For a planet located in a bright ring, the contrast gain is rather stable $(G \approx 10)$. For a planet located in a dark ring, the contrast gain fluctuates in between two regimes: no gain $(G \approx 1)$ and high gain $(G \approx 100-1000)$. Such regimes are smoothed far from the PSF centre. A planet at large separation rotates rapidly across many narrow high-gain zones having a width of approximately a degree. A planet at small separation rotates slowly across few large highgain zones with a width of typically few degrees. To probe several high-gain zones, a sufficient parallactic rotation rate is required especially for closer planets. Finally, the whole observation time is substantially reduced for a fast sky rotation when the star passes at its meridian, as requested for ADI.

\section{DISCUSSION}

\subsection{LBTI Fizeau as a direct imager}

From the imaging point of view, the LBTI in Fizeau mode is an interferometric direct imager. The interferometric PSF is directly suitable for direct imaging applications. The entrance aperture is made of two large subapertures of diameter $D=8.4 \mathrm{~m}$ having their centres separated by $B=14.4 \mathrm{~m}$ and a maximum base length of $B_{\max }=B+D=22.8 \mathrm{~m} .{ }^{1}$ The LBTI along its baseline can thus be compared to a very large $23-\mathrm{m}$ telescope with a 6-m central obscuration, performing the same resolution power in this direction.

We have shown a fundamental gain in contrast when combining coherently monochromatic light from two AO telescopes instead of using a single stand-alone AO telescope, assuming efficient control and acquisition systems at high speed. Instead of sampling the whole pupil of a very large telescope, probing the pupil only in one main direction enables high-contrast imaging. Indeed, aberrations inside a large 23-m telescope but outside of the two 8-m telescopes produces incoherent light that is not transmitted, avoiding additional speckles affecting the PSF contrast of the LBTI.

\subsection{One-directional interferometric sampling}

From the interferometric point of view, all the high-angular resolution information is sampled in the baseline direction. The $(\mathrm{u}, \mathrm{v})$ plane coverage is continuous in this direction, as shown in the modulation transfer function (Patru et al. 2017). The LBTI exploits solely two large subapertures with respect to a relatively small baseline $(B \approx D)$. On the contrary, the conventional non-redundant aperture masking technique (Lacour et al. 2011) relies on many small subapertures separated by long baselines $(B \gg D)$, which sample a finite number of distinct angular frequencies with various base lengths and orientations, in order to avoid angular frequency mixing in the presence of turbulence.

The LBTI aperture samples numerous angular frequencies in one main direction while removing the other frequencies in other directions. The angular frequency sampling is redundant with many identical baselines spanning two points in the entrance aperture and should suffer from frequency mixing due to the atmospheric turbulence. The different phases of redundant baselines are added incoherently and the angular frequency peaks are mixed by the interferometric combination. Such residual wavefront errors can be calibrated by means of techniques such as non-redundant aperture masking (Leisenring et al. 2012) or speckle masking interferometry with the LBTI (Reinheimer et al. 1997). Furthermore, the redundant frequencies can be added efficiently thanks to the highly coherent

\footnotetext{
${ }^{1}$ In practice, $D=8.36$ and $B_{\max }=22.65 \mathrm{~m}$, because the adaptive secondaries in each LBT are purposefully undersized to control the thermal background. The obscuration of the swing arm spiders supporting M2 and M3 has not been included here.
} 

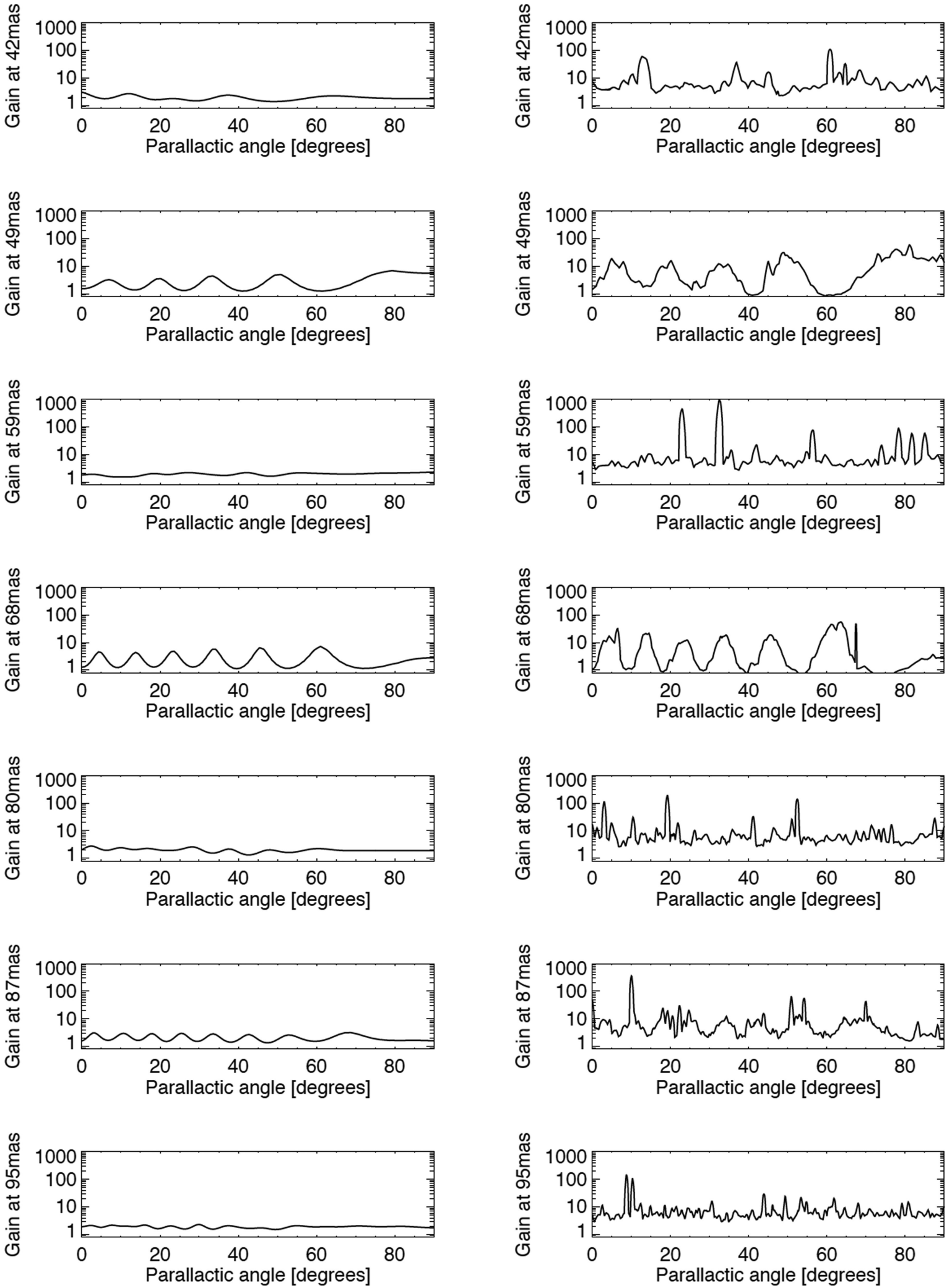

Figure 10. Contrast gain profiles of the dual versus single aperture depending on the parallactic angle over $90^{\circ}$ at several radial distances from the central axis, corresponding alternatively to a dark ring (31, 49, 68, 87 mas) and to a bright ring (42, 59, 80, 95 mas). Long exposures ratio $G_{\mathrm{LE}}$ (left) and averaged short exposures ratio $G_{\mathrm{SE}}$ (right). 
LBTI aperture corrected by AO and cophasing systems. In other words, the LBTI Fizeau imager performs a one-directional interferometric sampling by two large and close subapertures, for preserving in this direction a high-angular resolution and a sufficient $(\mathrm{u}, \mathrm{v})$ plane coverage, while improving the dynamic range across the AO-corrected field of view.

The fundamental gain in contrast provided by the LBTI facility should be exploited for future projects in interferometry. For instance, the Giant Magellan Telescope (GMT) could be used in Fizeau mode by alternatively changing the baseline among the ones provided by the seven primary mirrors, i.e. by transmitting on to the scientific channel only the beams coming from two AO-corrected mirrors at a time and by producing several interferometric PSFs with different fringes orientations. It would perform a better contrast than for classical imaging modes using simultaneously all the primary mirrors of the GMT.

\subsection{Independent correction of $\mathrm{AO}$ and piston errors}

$\mathrm{AO}$ and piston corrections are performed independently from side to side of the LBTI aperture split into two coplanar subapertures. Each AO system performs a wavefront correction across an 8-m pupil only. Consequently, the two AO secondaries of the LBT facility are smaller units that are easier to build than a complex AO system for a large 23-m telescope.

Against piston errors, the LBTI can rely on a fast camera to freeze the fringes in short exposures and/or a fringe tracker to compensate in real time the fringes displacements. The use of a fringe tracker is simplified by the fact that the LBTI is conceived as a pure Fizeau interferometer, without any need of complex delay lines, because the two entrance mirrors are coplanar. Otherwise, the cophasing requirements can be avoided and transposed to high frame rate requirements (for bright objects; Patru et al. 2017).

\subsection{LBTI Fizeau imager versus speckle interferometry}

Speckle imaging and speckle interferometry (Labeyrie 1970) exploit the diffraction limited resolution in snapshots against atmospheric turbulence. The principle for speckle imaging with a large telescope is that the minimum size of the speckles is equal to the size of the diffraction-limited Airy disc provided by the telescope. In addition for speckle interferometry using two large telescopes, two different speckle patterns are superposed and fine fringes are produced inside the speckles if the beams are coherent. Therefore, speckled - and fringed - snapshots contain more high-angular resolution information on frozen features than long exposure images having blurred features. However, such method also provides contrasted speckles only if the phase uniformity can be recovered in the pupil plane.

The same approach can be extrapolated to the contrast gain in the case of an AO-corrected interferometer. Similar to the speckle sizes getting closer to the diffraction limit, the maximum depth of the fringed speckles pattern is getting deeper by using the interferometer in a high-Strehl AO regime at high frame rate. The LBTI thus produces highly contrasted fringed speckles across the AO-corrected field of view. Whereas conventional speckle interferometry requires solely a fast camera, the LBTI uses AO systems for bringing the subapertures into a coherent state and, in addition, a cophasing system for compensating the piston unseen by both AO. Finally, AO speckle interferometry uses efficient AO and cophasing systems at high frame rate, enabling to freeze both speckles and fringes fluctuations (Patru et al. 2017). The fringed speckle halo in short exposures contains not only high-angular resolution information, as stated by speckle imaging and speckle interferometry, but also high-contrast imaging information.

\subsection{LBTI Fizeau sensitivity versus single aperture}

The limiting magnitude for guide stars and scientific targets should be determined for using efficiently the LBTI in Fizeau mode. First, poor atmospheric conditions and faint guide stars will seriously mitigate the AO performance, and thus the LBTI performance. Secondly, the fringe tracking control is also limited in performance when tracking on to a faint star. Thirdly, long exposures are required for faint science targets, preventing the ability to freeze the fringes and to preserve the contrast gain in the final PSF.

If the star is bright, we may require high-Strehl $\mathrm{AO}$ above 70 per cent, efficient cophasing system with piston $\mathrm{rms}$ lower than $\lambda / 8$ and exposure time shorter than the coherence time (Patru et al. 2017). A contrast gain of 10 - or more - can be achieved everywhere in the AO-corrected field of view. Providing narrow-band filters, a huge gain of up to $100-1000$ is further attainable in some locations in the dark features. Once such level of performance can be achieved on the LBTI in favourable conditions, a high parallactic rotation rate may be used to explore huge-gain zones and/or to develop an ADI-Fizeau mode. If the star is faint and/or if the atmospheric conditions are worse, we may rely on long exposures with moderate $\mathrm{AO}$ and cophasing performance, lowering the efficiency of such LBTI Fizeau mode (Patru et al. 2017).

The LBTI collects two times more flux than a stand-alone LBT and produces a fringe intensification doubling the maximum irradiance of the interferometric PSF (Section 2.1). Combining coherently the two subapertures in Fizeau mode instead of using them individually (e.g. in LEECH mode) (Skemer et al. 2014; Maire et al. 2015) improves the sensitivity by a factor of 4 . This makes the LBTI rather efficient as regards the point source sensitivity. In fact, we have restrained our study to bright and unresolved sources, but the extended source sensitivity of the LBTI can be dramatically affected in real conditions of observations.

Thanks to its closely spaced large elements and its well filled (u,v) plane in the baseline direction, the LBTI should have the sensitivity to image objects of low surface brightness, against the photon noise of the sky background (Angel et al. 1998). However, the source photon flux per resolution element is reduced by a factor $\left(2 D / B_{\max }\right)^{2}$, while the sky background photon flux remains essentially the same. The signal-to-noise ratio thus depends as $\left(D / B_{\max }\right)^{2}$, and the exposure time to reach given surface brightness increases as $\left(B_{\max } / D\right)^{4}$. While the resolution is improved by a factor of $B_{\max } / D=2.7$, the exposure time has to be increased by 50 times for the LBTI Fizeau interferometer compared to the single LBT aperture in order to image faint and extended targets (Angel et al. 1998).

\section{CONCLUSION}

The unique LBT facility with its LBTI instruments may reach unprecedented performance in sensitivity, resolution and dynamic range across the AO-corrected field of view. Compared to a single stand-alone 8-m LBT aperture, the 23-m LBTI Fizeau imager can provide a gain in sensitivity (by a factor of 4), a gain in angular resolution (by a factor of 3 ) and, as well, a gain in raw contrast (by a factor of 2-1000 varying over the AO-corrected field of view). We assert that the LBTI Fizeau imager can fundamentally provide a gain in contrast in comparison with current LBT modes using individual aperture. Such performance requires efficient control and 
acquisition systems at high speed (i.e. residual piston and $\mathrm{AO}$ rms compensation better than a fraction of the wavelength, coherence time of the turbulence shorter than the exposure time). The effects due to various sources of aberrations will be addressed subsequently, the AO and piston errors being explored in the next paper (Patru et al. 2017).

We stress that the significant contrast gain is rendered possible by the specific design of the LBTI Fizeau imager, including: (i) onedirectional interferometric sampling by two large and close subapertures instead of a very large aperture, (ii) independent correction of $\mathrm{AO}$ and piston errors from side to side of the LBTI aperture split into two coplanar subapertures and (iii) speckle imaging and speckle interferometry at high frame rate with efficient $\mathrm{AO}$ and cophasing systems, enabling to freeze both speckles and fringes fluctuations. We argue that the fringed speckle halo in short exposures contains not only high-angular resolution information, as stated by speckle imaging and speckle interferometry (Labeyrie 1970), but also highcontrast imaging information.

To go further, post-processing can be applied to enhance the image quality (e.g. by deconvolving each individual frame). A selection of high-quality frames should also be performed among the whole data set to improve the signal-to-noise ratio by using, for instance, merit functions (Patru et al. 2017). A drastic selection in a lucky imaging mode (Fried 1978) - would allow us to directly detect a compagnon located in a high-gain zone. In particular, we would recommend to adapt an ADI-like technique for searching extrasolar planets, taking advantage of the numerous high-contrast gain zones. Such high-gain zones are shown here in the contrast gain map defined as the ratio of the PSFs provided by the LBTI versus the LBT. On the contrary to the raw contrast gain defined here, the final contrast gain should account for data post-processing (e.g. by means of ADI). Finally, in a forthcoming paper, we will develop an endto-end simulation tool by modelling science cases (e.g. binary, exoplanet) and realistic aberrations (e.g. residual piston/tip-tilt fluctuations, wide-field AO errors, pointing errors, misalignments, spectral bandwidth) and by using post-processing techniques (e.g. frames selection, lucky imaging, shift-and-add, deconvolution, ADI mode).

This paper supports the development of a high-contrast interferometric mode of observation by using the LBTI in Fizeau mode compared to single aperture operation. An ADI-Fizeau mode seems right now feasible in the near-infrared wavelength, at least for standard science cases (Patru et al. 2017). It could be tested at the LBTI to complement the exoplanet survey LEECH in $L^{\prime}$ band (Skemer et al. 2014; Maire et al. 2015) and the HOSTS project (Danchi et al. 2016; Mennesson et al. 2016). Next, it should be extended to the visible wavelengths like the LIVE project (Hinz et al. 2014). Indeed, the visible PSF contains thinner structures than the nearinfrared PSF, providing numerous dark fringes and dark rings where exoplanets can be detected at low level. For a given field of view, the PSF has about 10 times more fringes and rings in the $I$ ' band $(\lambda=0.76 \mu \mathrm{m})$ than in the $L^{\prime}$ band $(\lambda=3.8 \mu \mathrm{m})$. The LBTI Fizeau imager can thus achieve high-angular resolution and high-dynamic range, outperforming the current class of 8-m telescopes.

\section{REFERENCES}

Agapito G., Puglisi A., Esposito S., 2016, in Marchetti E., Close L. M., Véran J.-P., eds, Proc. SPIE Conf. Ser. Vol. 9909, Adaptive Optics Systems V. SPIE, Bellingham, p. 99097E

Angel J. R. P., Woolf N., 1997, in Soderblom D., ed., ASP Conf. Ser. Vol. 119, Planets Beyond the Solar System and the Next Generation of Space Missions. Astron. Soc. Pac., San Francisco, p. 207
Angel J. R. P., Hill J. M., Strittmatter P. A., Salinari P., Weigelt G., 1998, in Reasenberg R. D., ed., Proc. SPIE Conf. Ser. Vol. 3350, Astronomical Interferometry. SPIE, Bellingham, p. 881

Danchi W. et al., 2016, in Malbet F., Creech-Eakman M. J., Tuthill P. G., eds, Proc. SPIE Conf. Ser. Vol. 9907, Optical and Infrared Interferometry and Imaging V. SPIE, Bellingham, p. 990713

Defrère D. et al., 2014, in Rajagopal J. K., Creech-Eakman M. J., Malbet F., eds, Proc. SPIE Conf. Ser. Vol. 9146, Optical and Infrared Interferometry IV. SPIE, Bellingham, p. 914609

Esposito S. et al., 2010, in Ellerbroek B. L., Hart M., Hubin N., Wizinowich P. L., eds, Proc. SPIE Conf. Ser. Vol. 7736, Adaptive Optics Systems II. SPIE, Bellingham, p. 773609

Fried D. L., 1978, J. Opt. Soc. Am., 68, 1651

Herbst T., 2003, Ap\&SS, 286, 45

Hill J. M., Ashby D. S., Brynnel J. G., Christou J. C., Little J. K., Summers D. M., Veillet C., Wagner R. M., 2014, in Stepp L. M., Gilmozzi R., Hall H. J., eds, Proc. SPIE Conf. Ser. Vol. 9145, Ground-based and Airborne Telescopes V. SPIE, Bellingham, p. 914502

Hinz P. et al., 2012, in Delplancke F., Rajagopal J. K., Malbet F., eds, Proc. SPIE Conf. Ser. Vol. 8445, Optical and Infrared Interferometry III. SPIE, Bellingham, p. 84450

Hinz P. et al., 2014, in Rajagopal J. K., Creech-Eakman M. J., Malbet F., eds, Proc. SPIE Conf. Ser. Vol. 9146, Optical and Infrared Interferometry IV. SPIE, Bellingham, p. 914605

Hinz P. M. et al., 2016, in Malbet F., Creech-Eakman M. J., Tuthill P. G., eds, Proc. SPIE Conf. Ser. Vol. 9907, Optical and Infrared Interferometry and Imaging V. SPIE, Bellingham, p. 990704

Labeyrie A., 1970, A\&A, 6, 85

Lacour S., Tuthill P., Amico P., Ireland M., Ehrenreich D., Huelamo N., Lagrange A.-M., 2011, A\&A, 532, A72

Leisenring J. M. et al., 2012, in McLean I. S., Ramsay S. K., Takami H., eds, Proc. SPIE Conf. Ser. Vol. 8446, Ground-based and Airborne Instrumentation for Astronomy IV. SPIE, Bellingham, p. 84464F

McCarthy D. W., Sabatke E. M., Sarlot R. J., Hinz P. M., Burge J. H., 2000, in Léna P. J., Quirrenbach A., eds, Proc. SPIE Conf. Ser. Vol. 4006, Interferometry in Optical Astronomy. SPIE, Bellingham, p. 659

Maire A.-L. et al., 2015, A\&A, 576, A133

Marois C., Lafrenière D., Doyon R., Macintosh B., Nadeau D., 2006, ApJ, 641,556

Mennesson B. et al., 2016, Malbet F., Creech-Eakman M. J., Tuthill P. G., eds, Proc. SPIE Conf. Ser. Vol. 9907, Optical and Infrared Interferometry and Imaging V. SPIE, Bellingham, p. 99070X

Noll R. J., 1976, J. Opt. Soc. Am., 66, 207

Patru F., Esposito S., Puglisi A., Riccardi A., Pinna E., Arcidiacono C., Hill J., Hinz P., 2016a, in Malbet F., Creech-Eakman M. J., Tuthill P. G., eds, Proc. SPIE Conf. Ser. Vol. 9907, Optical and Infrared Interferometry and Imaging V. SPIE, Bellingham, p. 99071S

Patru F., Esposito S., Puglisi A., Riccardi A., Pinna E., Arcidiacono C., Hill J., Hinz P., 2016b, in Malbet F., Creech-Eakman M. J., Tuthill P. G., eds, Proc. SPIE Conf. Ser. Vol. 9907, Optical and Infrared Interferometry and Imaging V. SPIE, Bellingham, p. 99071T

Patru F., Esposito S., Puglisi A., Riccardi A., Pinna E., Arcidiacono C., Hill J., Hinz P., 2017, Opt. Commun., MNRAS, in press

Reinheimer T., Hofmann K.-H., Scholler M., Weigelt G., 1997, A\&AS, 121, 191

Skemer A. J. et al., 2014, in Marchetti E., Close L. M., Véran J.-P., eds, Proc. SPIE Conf. Ser. Vol. 9148, Adaptive Optics Systems IV. SPIE, Bellingham, p. 91480L

This paper has been typeset from a $\mathrm{T}_{\mathrm{E}} \mathrm{X} / \mathrm{LT} \mathrm{E} \mathrm{X}$ file prepared by the author. 